\title{
Evaluation of PHB/Clay Nanocomposite by Spin-Lattice Relaxation Time
}

\author{
Mariana Bruno, Maria Inês Bruno Tavares*, Leandro Medeiros Motta, Eduardo Miguez, \\ Monica Preto, Amanda Oliveira Rodriguez, Fernandez \\ Centro de Tecnologia, Instituto de Macromoléculas Professora Eloisa Mano - IMA, \\ Universidade Federal do Rio de Janeiro - UFRJ, \\ Bloco J, Ilha do Fundão, CP 68525, 21945-970 Rio de Janeiro - RJ, Brazil
}

Received: September 27, 2008; Revised: December 1, 2008

\begin{abstract}
Poly(3-hydroxybutyrate) (PHB) based on nanocomposites containing different amounts of a commercial organically modified clay (viscogel B7) were prepared employing solution intercalation method. Three solvents, such as: $\mathrm{CHCl}_{3}$, dimethylchloride (DMC) and tetrahydrofuran (THF) were used. The relationship among the processing conditions; molecular structure and intermolecular interaction, between both nanocomposite components, were investigated using a nuclear magnetic resonance (NMR), as a part of characterization methodology, which has been used by Tavares et al. It involves the hydrogen spin-lattice relaxation time, $\mathrm{T}_{1} \mathrm{H}$, by solid state nuclear magnetic resonance, employing low field NMR. X ray diffraction was also employed because it is a conventional technique, generally used to obtain the first information on nanocomposite formation. Changes in PHB crystallinity were observed after the organophilic nanoclay had been incorporated in the polymer matrix. These changes, in the microstructure, were detected by the variation of hydrogen nuclear relaxation time values and by X ray, which showed an increase in the clay interlamelar space due to the intercalation of the polymer in the clay between lamellae. It was also observed, for both techniques, that the solvents affect directly the organization of the crystalline region, promoting a better intercalation, considering that they behave like a plasticizer.
\end{abstract}

\section{Keywords: $N M R, P H B$, Nanocomposite}

\section{Introduction}

Biodegradable polymers have been focused on many studies, due to the fact that the study of these polymers is a topic of high interest because of the great quantity of wasted polymers in the world. The development of biodegradable materials with controlled properties has been widely studied. PHB is one of the most studied biodegradable polymers used to substitute synthetic plastics in their application that causes an environmental impact, such as food packaging. Therefore, the use of biodegradable polymers is limited due to its characteristics such as molecular weight; structural crystallinity and amorphous phase, as well as its narrow range of processing temperature ${ }^{1,2}$; these characteristics promote bad mechanical properties. According to these statements some modifications in the chemical structure of these biopolymers can be done. Copolymerization with valerate generates a poly-b-(hydroxybutyrate-co-valerate) (PHB-HV), which is one way to improve the PHB mechanical properties. PHB-HV is less stiff and tougher than PHB and because of this the copolymer is used as packaging material. Then, the generation of nanocomposite based on the biodegradable polymers has been considered one of the best routes to improve the mechanical properties of $\mathrm{PHB}^{3,4}$.

The preparation of hybrid nanocomposite, employing inorganic nanofiller, such as montmorillonite and/or other type of organically modified clay, has a great industrial interest to produce biodegradable nanocomposites that has its crystallinity reduced; gas and water vapor barrier increased; as well as an increase in the thermal stability ${ }^{3-5}$.

To accompany these studies some conventional techniques, such as thermal analyzes, $\mathrm{X}$ ray diffraction and transmission electronic microscopy (TEM) are normally used. Therefore, one of the best spectroscopy that has been initiated in these studies is nuclear magnetic resonance, which is able to evaluate the polymer samples in solution and solid state ${ }^{5-8}$. The solid state techniques can include the hydrogen nuclear relaxation measurements, which will give response on the molecular dynamic of the hybrid compound and it also evaluates the nanoparticle dispersion in the polymer matrix $x^{3,4,9,10}$. The hydrogen relaxation times s,10,13,14 $^{9}$ can be measured directly in a low field NMR and it gives response on the nanoparticle dispersion; interaction between both nanocomposite components and better processing conditions $\mathrm{s}^{9,10}$.

In this work nanocomposites based on PHB, containing 1, 3 and 5 wt. (\%) of commercial organo-modified montmorillonite clay (viscogel B7 - which is intercalated with C-12) were prepared by solution intercalation reaction and they were characterized using a methodology (developed in our laboratory ${ }^{9-10}$ ), which involves the determination of hydrogen spin-lattice relaxation time, by nuclear magnetic resonance, and the use of conventional $\mathrm{X}$ ray diffraction to measure the basal space of nanoclay. Both techniques used give information that is complementary, because they are measured in different time scale.

In this work we have decided not to use transmission electronic microscopy (TEM), due to the fact that the micrographs do not represent the median behavior of the sample, as NMR do. Besides the quantity of sample analyzed by TEM is very small and it is not representative comparing to $5 \mathrm{~g}$ used in the NMR measurements.

\section{Experimental}

The nanocomposites were prepared by solution intercalation reaction, which is a non aggressive method, using $\mathrm{CHCl}_{3}$, dimethylchloride (DMC) and tetrahydrofuran (THF) as solvents. These sol- 
vents were chosen according to their solubility obtained by previous study in PHB. The solutions of PHB and organoclay were prepared isolated to obtain the polymer/clay ratios $99 / 1,97 / 3$ and $95 / 5$ percentage of clay in the polymer matrix. The isolated solutions were kept in the agitation for 24 hours at room temperature. After that, the solutions were mixed and left for 24 hours being stirred. At the end of this time the solutions were casting on the plates and were kept at room temperature. These conditions were careful chosen, taking into consideration that PHB is a biodegradable polymer with singular properties and characteristics.

$\mathrm{X}$ ray diffraction (XRD) analyses were done in a Rigaku $\mathrm{D} / \mathrm{max}$ 2400 diffractrometer with nickel-filtered $\mathrm{CuK} \alpha$ radiation of wavelength $1.542 \AA$, at room temperature scanning over angle $(2 \theta)$ range from $2^{\circ}$ to $20^{\circ}$ in 0.02 steps, at a scanning rate of $8 \% \mathrm{~min}$.

Low field NMR spectrometer, MARAN ultra 23 (Resonance Oxford - UK), operating at $23 \mathrm{MHz}$ (for hydrogens) and equipped with an $18 \mathrm{~mm}$ variable temperature probe, was used for the hydrogen relaxation measurements. Hydrogen spin-lattice relaxation times were determined directly by the traditional inversion-recovery pulse sequence (recycle delay - $180^{\circ}-\tau-90^{\circ}$ - acquisition), the $90^{\circ}$ pulse, $4.6 \mu \mathrm{s}$, was calibrated automatically by the instrument software. The amplitude of the FID was sampled for twenty $\tau$ data points, ranging from 0.1 to $5000 \mathrm{~ms}$, with 4 scans each and 5 seconds of recycle delay. The relaxation values and relative intensities were obtained by fitting the exponential data with the aid of the WINFIT (program supplied from the Resonance).

\section{Results and Discussion}

The casting films of the nanocomposites, prepared in different solvents (DMC, THF and $\mathrm{CHCl}_{3}$ ), were analyzed by $\mathrm{X}$ ray diffraction and the profiles obtained for each nanocomposite are shown in Figures from 1 to 3 , respectively.

The disappearance of the main peak of the clay, which shows the intensity of lamellae organization (at 2 $\theta$ ), can be attributed to the nanocomposites formation. Although, for the nanocomposites prepared in THF, the referring peak of the clay did not disappear completely, this can indicate that this is not a good solvent for this system. Comparing widths of the half height of the nanocomposites prepared in DMC and chloroform, one concludes that both solvents are good for the preparation of nanocomposites $\mathrm{PHB} / \mathrm{clay}$, due to the intercalation/exfoliation formation. One can observe that the decreasing in the peaks intensity is an indication of the increase in the exfoliation process, especially when $5 \%$ of nanoclay was incorporated. However, a non similar behavior was found for the nanocomposites prepared in THF, which can be attributed to a no good solubilization/interaction of the system in this solvent. Knowing that the $\mathrm{X}$ ray scale is not enough to obtain more information than it was explored, we have decided to use solid state NMR, which is more sensitive and permits to evaluate the median behavior of the sample, because the time scale of the measurement is at the molecular level and the domains detected have sizes, which vary from 25 to $50 \mathrm{~nm}$.

According to the main purpose of this work, hydrogen spin-lattice relaxation time measurements were recorded to get more information on samples homogeneity; clay dispersion and interaction process in the nanocomposite. The relaxation data measured from one fit exponential are listed in the Table 1.

The evaluation of the relaxation data obtained from the one fit exponential reveals the major contribution of the domain that controls the relaxation process. In solids the hydrogen spin-lattice relaxation is controlled by the dipolar intermolecular interaction process. From Table 1 the results showed that DMC was a better solvent for the inter-

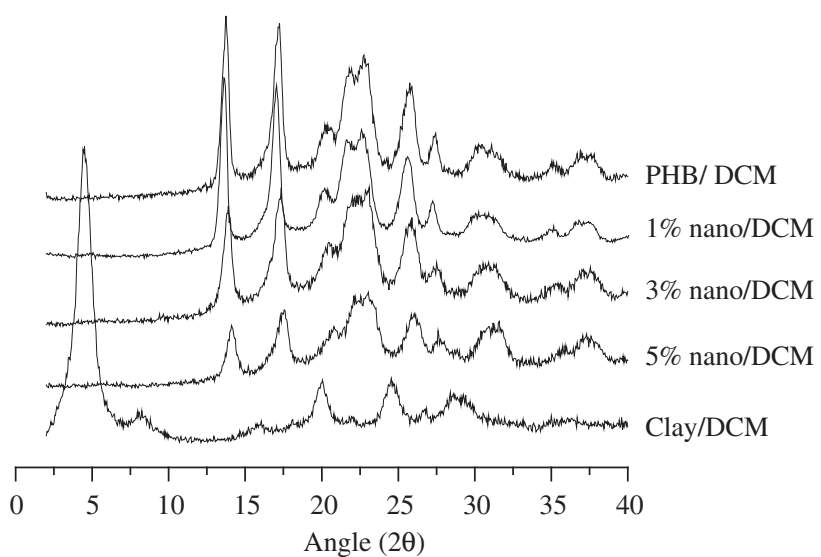

Figure 1. X ray diffraction of the nanocomposites prepared in DMC.

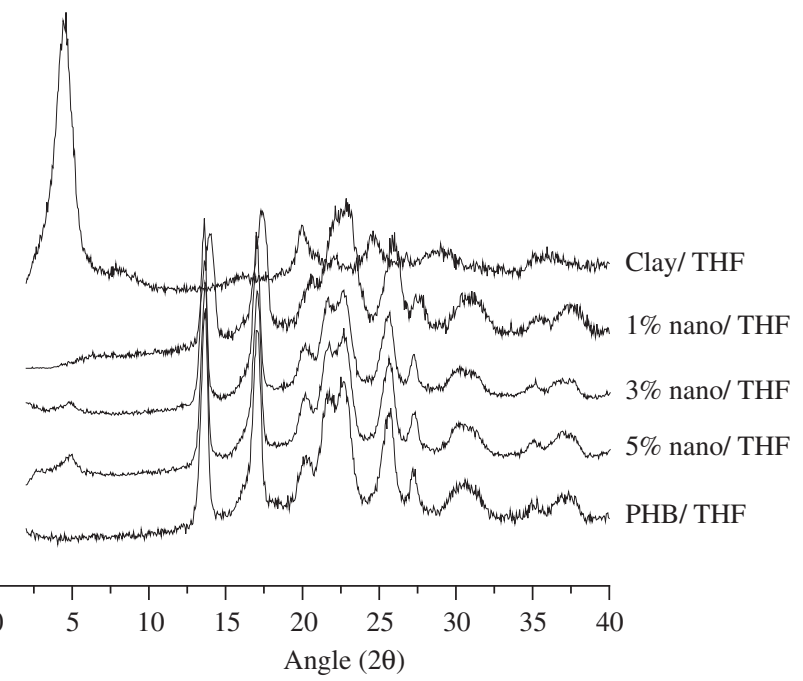

Figure 2. $\mathrm{X}$ ray diffraction of the nanocomposites prepared in THF.

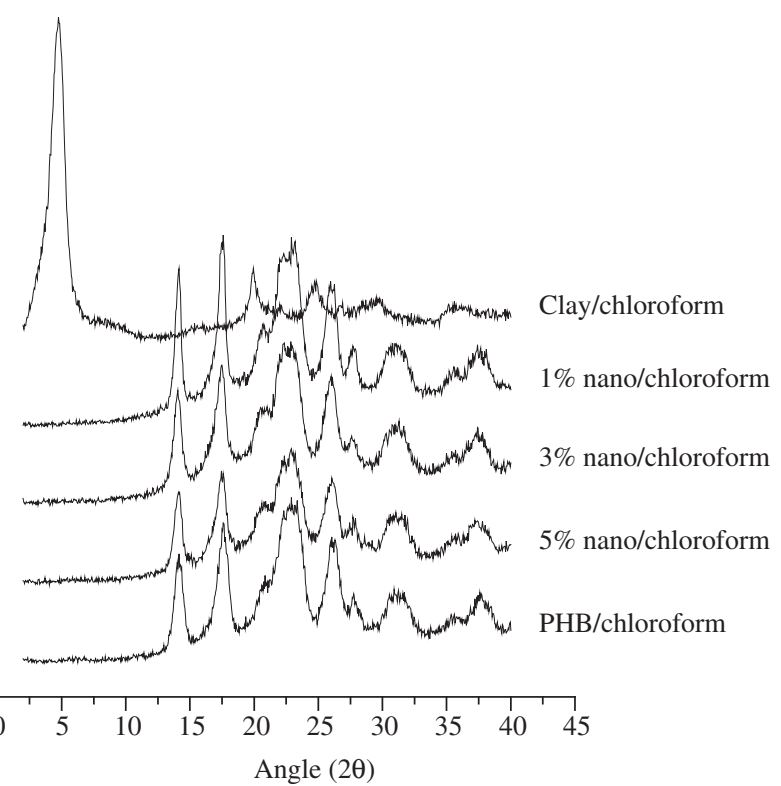

Figure 3. $\mathrm{X}$ ray diffraction of the nanocomposites prepared in $\mathrm{CHCl}_{3}$. 
Table 1. $\mathrm{T}_{1} \mathrm{H}$, obtained with one fit data for the nanocomposites.

\begin{tabular}{lc}
\hline \multicolumn{1}{c}{ Sample } & $\mathrm{T}_{1} \mathrm{H}_{\text {onefit }}(\mathrm{ms})$ \\
\hline PHB (DMC) & 600 \\
PHB/clay 1\%(DMC) & 456 \\
PHB/clay 3\% (DMC) & 451 \\
PHB/clay 5\% (DMC) & 500 \\
& \\
PHB (THF) & 513 \\
PHB/clay 1\% (THF) & 471 \\
PHB/clay 3\% (THF) & 459 \\
PHB/clay 5\% (THF) & 500 \\
& \\
PHB (CHCl $)$ & 563 \\
PHB/clay 1\% $\left(\mathrm{CHCl}_{3}\right)$ & 451 \\
PHB/clay 3\% $\left(\mathrm{CHCl}_{3}\right)$ & 474 \\
PHB/clay 5\% $\left(\mathrm{CHCl}_{3}\right)$ & 441 \\
\hline
\end{tabular}

calation/exfoliation process. First of all the difference from the PHB relaxation value and the values determined for the nanocomposite is higher than the other two solvents, showing that in DMC a good solubilization of both nanocomposite components was found. Another point is related to the fact that no difference between $\mathrm{T}_{1} \mathrm{H}$ values was found for 1 and $3 \%$ of nanoclay incorporated in the PHB matrix. This fact can indicate that the clay had a very good dispersion and a good exfoliated/intercalated nanocomposite ratio was also found. Besides this, a pronounced change, which is an increase in the relaxation data, was detected when $5 \%$ of nanoclay was incorporated in relation to the value of PHB in this solvent. This can be explained as an increase in the intercalation, because an exfoliation process causes a decrease in the value of hydrogen relaxation time, comparing to the initial polymer, since the polymer chains are around the lamellae, and the metals present in the lamellae promote a decreasing in the relaxation time of the nucleus near them. However, an increase in the hydrogen relaxation when $5 \%$ of nanoclay was introduced indicates that more intercalated nanocomposite was formed, because the intercalation process restricts the polymer chains molecular mobility, which is reflected in the hydrogen relaxation value. According to the data both 1 and $3 \%$ of nanoclay were very well dispersed in DMC solvent.

Analyzing the hydrogen relaxation data for the nanocomposite prepared through THF, one can see that for both 1 and $3 \%$ of nanoclay there was a decrease in the relaxation values, less pronounced than for DCM, and the same numeric value of relaxation was found for 5\% of nanoclay. However, the difference between the value of relaxation for PHB and nanocomposite with $5 \%$ is practically zero, which is an indication that this quantity of nanoclay was not good dispersed and no interaction between the nanocomposite components was found for this ratio. These results confirm the morphological behavior found by $X$ ray diffraction, for the $5 \%$ of nanoclay, the peak in $2 \theta$ is still there. In THF $5 \%$ of nanoclay was not well dispersed and no PHB nanocomposite was formed. According to the relaxation results both 1 and $3 \%$ of nanoclay were better dispersed in solvent, but $3 \%$ of its behavior was a little better.

Evaluating the nanocomposites obtained by chloroform, for all nanoclay ratios, there was a decrease in the relaxation values and no significant change was observed in the values of hydrogen relaxation time for all nanoclay ratios, comparing to PHB. This fact is an indication that this solvent also promotes an intercalation and/or exfoliation, due to the similarity in the relaxation parameter which comes from the fact that $\mathrm{CHCl}_{3}$ promotes a different organization of the nanocomposite that is independent from nanoclay ratio. This particularity can be attributed to the interaction forces of the solvent solubilization. Analyzing the hydrogen relaxation value for $5 \%$ of nanoclay, it can be concluded that it is a better ratio for this solvent.

\section{Conclusions}

According to the results, one can conclude that the methodology employed was efficient, rapid and practical to better understand the behavior of the nanocomposites formed by PHB/nanoclay. Both $\mathrm{X}$ ray and relaxation time techniques were complementary. The NMR relaxation data informed about the nanoclay dispersion; interaction; nanocomposite structure organization, and also inferred about the better nanoclay ratio for each solvent.

\section{Acknowledgements}

The authors thank you CNPq and FAPERJ for the support of this work.

\section{References}

1. Rosa DS, Lotto NT, Loppes DR, Guedes CG. The use of roughness for evaluating the biodegradation of poly-b-(hydroxybutirate) and poly-b(hydroxybutirate-co-b-valerate). Polymer Testing 2004; 23(1): 3-8.

2. Rosa DS, Calil MR, Guedes CGF, Rodrigues TC. Biodegradability of Thermally Aged PHB, PHB-V and PCL in Soil Compostage. Journal of polymers and the environment 2004; 12(4): 239-245.

3. Bruzaud S, Bourmaud A. Thermal degradation and (nano) mechanical behavior of layered silicate reinforced poly(3-hydroxybutyrate-co3-hydroxyvalerate) nanocomposites. Polymer Testing 2007; 26(5): 652-659.

4. Okamoto M. Biodegradable polymer/layered silicate nanocomposites: a review. In: Handbook of Biodegradable Polymeric Materials and their Application. Surya Mallapragada and Balaji Narasimahn; 2005: 1-45.

5. Kawasumi M, Hasegawa N, Kato M, Usuki A, Okada A. Preparation and Mechanical Properties of Polypropylene-Clay Hybrids. Macromolecules 1997; 30(20): 6333-6340.

6. Ray SS, Okamoto M. Polymer/layered silicate nanocomposites: a review from preparation to processing. Progress in Polymer Science 2003; 28(11): 1539-1641.

7. Nogueira RF, Tavares MIB, San Gil RAS. Carbon-13 Solid state NMR study od polypropylene/Clay Nanocomposite. Journal of Metastable and Nanocrystalline Materials 2004; 22:71-76.

8. Nogueira RF, Tavares MIB, San Gil RAS, Silva NM. Solid state NMR investigation of polypropylene/ brazilian clay blending process. Polymer Testing 2005; 24(2): 358-362.

9. Tavares MIB, Nogueira RF, San Gil RAS, Silva NM, Preto M, Rodrigues TC, Silva EO. Polypropylene-Clay Nanocomposite Structure probed by HNMR Relaxometry. Polymer Testing 2007; 26(6): 1100-1102.

10. Tavares MIB, Silva EO, Nogueira JS. Preparation and characterization of Nanocomposites Natural Resin of Amescla/MMT. In: Macro 2006 - World Polymer Congress / 41st International Symposium in Macromolecules; 2006; Rio de Janeiro; 2006.

11. Okada A, Usuki A. The Chemistry of Polymer-Clay hybrids. Materials Science and Engineering 1995; C(3): 109-113.

12. Kylest RE, Tonelli AE. Conformational Characteristics of poly (D- $\beta$ hidorxybutirate). Macromolecules 2003; 36(4): 1125-1131.

13. Conte P, Spaccini P, Piccolo A. State of the art of CPMAS ${ }^{13}$ C-NMR spectroscopy applied to natural organic matter. Progress in Nuclear Magnetic Resonance Spectroscopy 2004; 44(2): 215-221.

14. VanderHart DL, Asano A, Gilman JW. Solid State NMT investigation of paramagnetic Nylon-6 clay Nanocomposites. 1. Crystallinity,

15. Morphologya and the Direct Influence of $\mathrm{Fe}^{3+}$ on nuclear spin. Chemistry of Materials 2001; 13(11): 3781-3795. 